\title{
Reduction of Nonlinear Distortion in Directly Modulated Semiconductor Lasers by Coherent Light Injection
}

\author{
Gnitabouré Yabre and Jean Le Bihan, Fellow, IEEE
}

\begin{abstract}
A theoretical investigation of the second- and thirdorder intermodulation distortions (IMD's) in an injection-locked semiconductor laser under small-signal modulation is presented. The results show that a substantial reduction of the laser nonlinearity can be obtained, depending on both the injection level and frequency detuning between the master and slave lasers. The intensity modulation frequency response is also reported and shows that the injection-locked laser may also have a significantly improved behavior over the same free-running laser, revealed mostly in the reduced resonance peak and the broadening of the modulation bandwidth available.
\end{abstract}

Index Terms - Bandwidth, direct modulation, distortion, injection-locking, semiconductor lasers.

\section{INTRODUCTION}

$\mathbf{S}$ EMICONDUCTOR lasers have proven their usefulness in the field of optical fiber communications. Most of the systems developed so far use digital modulation, because of lower carrier-to-noise ratio needed in these modulation formats. However, since in the last ten years, analog modulation systems incorporating semiconductor lasers are emerging as an attractive field of application of these sources. Analog information can be upconverted to a narrow-band channel at high frequency, and multicarrier analog signals used in CATV, satellite, or radar systems can be simultaneously transmitted with the laser, fiber, and detector. Up to the present, most of the developed systems use the intensity modulation of the lasers and the direct detection of the emitted light. These techniques require that the laser light intensity be a perfectly linear function of the drive current, under modulation. But, due to the inherent nonlinearities of the lasers, energy is more or less transferred on parasitic frequencies which are all possible combinations of the original frequencies. These are undesirable harmonics and intermodulation products (IMP's). Such parasitic components in the output signal of a transmitter are of importance and great interest because they can limit the performance of the overall system. Although care is taken in designing analog laser sources, the problem of their nonlinearities is still a matter of question.

For low-frequency modulation like in cable television systems, the distortion is among other things caused by the

Manuscript received January 16, 1996; revised December 3, 1996.

The authors are with the Ecole Nationale d'Ingénieurs de Brest, Laboratoire RESO, Technopôle Brest-Iroise, 29608 Brest Cedex, France.

Publisher Item Identifier S 0018-9197(97)04718-0. nonlinear light-versus-current curve, which may be mainly due to leakage currents. In high-frequency modulation systems, which operate in multigigahertz range near the resonance frequency of the laser diodes, intrinsic dynamic nonlinearities will dominate [1]. These dynamic nonlinearities result from the interaction between photons and electrons in the laser cavity. They are well described by the rate equations. Previous publications have reported analytical expressions for the harmonics and IMD's in laser diodes using a perturbation analysis. Careful measurements have shown agreement with the theoretical predictions [2]-[5]. Another method of deriving the distortion characteristics of laser diodes is based on equivalent circuit modeling of the active region [6], [7]. Volterra functional series approach can also be used [8], [9] or a limited expansion based on the Bessel function [10], [11]. The Bessel function method has the main interest to be usable in the case of large-signal modulation. An advantage of the circuit model and Volterra series analysis is their applicability to any arbitrary input waveform. The circuit model also allows the implementation of available simulation software packages such as SPICE or MDS.

Regardless of the method employed to describe the dynamic behavior of laser diodes, the different studies have pointed out that the distortion levels can be substantially high, even at moderate optical modulation depths (OMD's), particularly at high modulation frequencies. The second harmonic distortion levels, for example, have been found to be higher than $-10 \mathrm{dBc}$ (dB with respect to the carrier) [3], at modulation frequencies near the half resonance frequency. This could lead to interchannel interference if channels are distributed over a bandwidth greater than one octave. So far, for some wideband applications, the use of linearization schemes has become necessary in order to reach the linearity requirements.

A number of techniques have been proposed to linearize optical transmitters. Electrooptical feedback or feedforward compensation techniques can be used in narrow-band systems [12], [13]. But each of these methods suffers from specific limitations. The main problem with feedback is that the loop delay must be very small. The main impairment of feedforward compensation is the difficulty of generating an accurate error signal. Another solution is based on predistortion, a method that consists of passing the modulation current through an adequate nonlinear element such that when the modified input signal is applied to the laser, an undistorted output results [14]. 
The main disadvantage with predistortion is the complexity of the electrical circuit to be designed and the difficulty of controlling the harmonics and IMD's at the same time.

As of this date, none of the above alternatives has taken the lead, although they have allowed a slight improvement of the laser linearity. As it is previously stated, their technical implementation appears most difficult. These different linearization techniques remain to be developed and improved. In this paper, a new method is proposed, where one directly injects a light beam from a CW laser (master) into the cavity of the modulated laser (slave), through an optical isolator.

The behavior of laser oscillators under the influence of an external light has been investigated by a number of authors. It has been shown, theoretically and experimentally, that such a type of operation affects the power and spectral characteristics of the injected laser. The four-wave mixing regime is well known and may be developed into a technique for optical-frequency conversion [15] or else for an accurate characterization of many intrinsic laser parameters [16]. Under light injection, other interesting phenomena, such as locked or unstable regimes may be observed with different operating conditions. Locking occurs when the optical frequency of the injected light is chosen within the so-called locking range [17], [18]. This is the range over which the frequency of the slave laser can be tuned while still locked to the master laser frequency. Once a perfect locking is reached, all power of the slave laser is emitted at the optical frequency of the master laser. If the injection conditions are fixed outside the locking range or if the external light is not strong enough to affect locking, then the slave laser may operate in an unstable or a four-wave mixing regimes.

The locking regime of an injected semiconductor laser is of great interest and may lead to practical applications in the area of telecommunications. Locking technique can be used, for example, for microwave signal generation [19] or chirp reduction in high-speed modulation systems [17]. In this paper, we have studied the IM characteristics of a high-speed laser diode under injection-locking and capable of being modulated at multigigahertz frequencies. The theoretical development consists of investigating the harmonic frequencies and IMP's generated, under a two-tone current modulation. The present analysis is based on the perturbation method applied to the rate equations. Semi-analytical expressions for the second- and third-order IMD's of the laser diode locked by an external light injection are derived and simulated. The transfer function is also reported. The curves are successfully compared with those of the same laser without light injection. To our knowledge, this work is the first demonstration of reduced nonlinear distortion in semiconductor lasers by injection-locking.

\section{THEORY}

The theoretical model used to describe a semiconductor laser with coherent light injection is based on the singlemode rate equations. In the literature, these equations often appear under various forms because of different parameter notations or normalizations and because of such and such physical phenomenon considered or not. Here, we adopt the formulation using the normalized form of [11]

$$
\begin{gathered}
\frac{d s}{d \tau}=\gamma\left[n_{\mathrm{th}} g(n, s) s-s+n_{\mathrm{th}} \beta n\right. \\
\left.\quad+2 \frac{\tau_{p}}{T}\left(\frac{s_{i}}{s}\right)^{1 / 2} \cos \theta s\right] \\
\frac{d n}{d \tau}=j-g(n, s) s-n \\
\frac{d \theta}{d \tau}=\gamma\left\{2 \pi \tau_{p} \Delta \nu_{0}-\frac{\alpha}{2}\left[n_{\mathrm{th}} g(n, s)-1\right]\right. \\
\left.\quad-\frac{\tau_{p}}{T}\left(\frac{s_{i}}{s}\right)^{1 / 2} \sin \theta\right\}
\end{gathered}
$$

with

$$
g(n, s)=\frac{1}{n_{\mathrm{th}}}\left(n_{\mathrm{th}} n+1-n_{\mathrm{th}}\right)\left(1-\varepsilon_{n} s\right)
$$

where $s$ and $n$ are, respectively, the normalized photon and electron densities, $j$ is the normalized injection current, $\tau$ is the normalized time, $\varepsilon_{n}$ is the normalized gain compression factor, $\gamma=\tau_{e} / \tau_{p}$ is the ratio between the electron lifetime and the photosion coupled to the lasing mode, $n_{\mathrm{th}}$ is the normalized carrier density at threshold, $\Delta \nu_{0}=\nu_{i}-\nu_{m 0}$ is the frequency detuning between the master laser and the injected mode of the slave one, $\theta=\varphi_{i}-\varphi$ is the phase detuning, $\alpha$ is the linewidth enhancement factor, $T=2 L / v_{g}$ defines the cavity round-trip time, $v_{g}$ being the group velocity of light and $L$ the cavity length, and $g(n, s)$ is the normalized optical gain.

For an injection current $j$, comprising the steady-state bias current $j_{0}$ and a small variation $j_{1}$, the photon and electron densities, and phase detuning are assumed to be perturbed by small amounts $s_{1}, n_{1}$, and $\theta_{1}$ around their steady-state values $s_{0}, n_{0}$, and $\theta_{0}$, that is, $j=j_{0}+j_{1}, s=s_{0}+s_{1}, n=n_{0}+n_{1}$, and $\theta=\theta_{0}+\theta_{1}$, where $j_{0} \gg j_{1}, s_{0} \gg s_{1}, n_{0} \gg n_{1}$, and $\theta_{0} \gg \theta_{1}$, in accordance with the small-signal analysis.

When the small-signal modulation current is a quasisinusoidal current with normalized angular frequencies $\omega_{1}=2 \pi \tau_{e} f_{1}$ and $\omega_{2}=2 \pi \tau_{e} f_{2}$, so that

$$
j_{1}=\sum_{\omega=\omega_{1}, \omega_{2}} \frac{1}{2}\left(j_{\omega} e^{i \omega \tau}+j_{\omega}^{*} e^{-i \omega \tau}\right)
$$

where $j_{\omega_{i}}^{*}$ represents the complex conjugate of $j_{\omega_{i}}$, the Fourier series expansion approach allows to express the perturbations $s_{1}, n_{1}$, and $\phi_{1}$ (with $\phi_{1}=2 s_{0} \theta_{1}$ ) under the following forms

$$
\begin{aligned}
& s_{1}=\sum_{\omega} \frac{1}{2}\left(s_{\omega} e^{i \omega \tau}+s_{\omega}^{*} e^{-i \omega \tau}\right) \\
& n_{1}=\sum_{\omega} \frac{1}{2}\left(n_{\omega} e^{i \omega \tau}+n_{\omega}^{*} e^{-i \omega \tau}\right) \\
& \phi_{1}=\sum_{\omega} \frac{1}{2}\left(\phi_{\omega} e^{i \omega \tau}+\phi_{\omega}^{*} e^{-i \omega \tau}\right) .
\end{aligned}
$$

The quantities $s_{\omega}, n_{\omega}$, and $\phi_{\omega}$ are the coefficients of the Fourier series expansion of the normalized photon and electron densities and phase detuning. The $\omega$ 's denote all the frequencies generated by the mixing of the carriers inside the laser diode, i.e., the fundamental frequencies $\omega_{i}(i=1,2)$, the harmonic frequencies $\left(2 \omega_{i}, 3 \omega_{i}, \cdots\right.$, as well as the IMP's at $\omega_{2} \pm \omega_{1}, 2 \omega_{1} \pm \omega_{2}$, and $\left.2 \omega_{2} \pm \omega_{1} \cdots\right)$, the general form of which is $\omega=m_{1} \omega_{1}+m_{2} \omega_{2}\left(m_{1}, m_{2} \in Z / \omega>0\right)$; the number 
$\left|m_{1}\right|+\left|m_{2}\right|$ represents the order of $\omega$, that is, the order of the signal component at angular frequency $\omega$. As outlined before, only the frequency response and the distortion characteristics due to the intermodulation products $\omega_{2}+\omega_{1}$ and $2 \omega_{1}-\omega_{2}$ will be illustrated. For the following, we shall find it convenient to denote $\omega_{2}+\omega_{1}, 2 \omega_{1}-\omega_{2}$ by $\omega_{I I}$ and $\omega_{I I I}$, respectively.

Under the above considerations, (1)-(3) gives a set of linearized equations in the frequency domain for $s_{\omega}, n_{\omega}$, and $\phi_{\omega}$, depending on $j_{\omega_{i}}$ and higher order terms which are combinations of $s_{\omega}, n_{\omega}$, and $\phi_{\omega}$. The obtained formulas can be condensed into the following matrix representation:

$$
\begin{gathered}
{\left[\begin{array}{ccc}
i \omega+a_{11} & a_{12} & a_{13} \\
a_{21} & i \omega+a_{22} & a_{23} \\
a_{31} & a_{32} & i \omega+a_{33}
\end{array}\right]\left[\begin{array}{c}
s_{\omega} \\
n_{\omega} \\
\phi_{\omega}
\end{array}\right]} \\
=\left(b_{\omega_{1}}^{m_{1} \mid} b_{\omega_{2}}^{\left|m_{2}\right|}\right)^{k_{m}}\left[\begin{array}{c}
Q_{s, \omega} \\
Q_{n, \omega} \\
Q_{\phi, \omega}
\end{array}\right]
\end{gathered}
$$

where the definition $k_{m}=0$ if $\left|m_{1}\right|+\left|m_{2}\right|=1$ and $k_{m}=1$ if $\left|m_{1}\right|+\left|m_{2}\right|>1$ has been introduced as well as the definition $b_{\omega_{i}}=s_{\omega_{i}}$ if $m_{i}>0$ and $b_{\omega_{i}}=s_{\omega_{i}}^{*}$ if $m_{i}<0$. The set of $a_{i j}$ are the elements of a matrix $A$ of order 3, depending only on the physical and injection parameters and the bias point of the laser. The detailed expressions of $a_{i j}$ are given in the Appendix. The quantities $Q_{s, \omega}, Q_{n, \omega}$, and $Q_{\phi, \omega}$ are the driving terms that must be specified to determine the solutions of (9)

The driving terms in (9) are calculated by successive approximations, in accordance with the small-signal analysis. We assume the following hypothesis: the amplitudes of the fundamental terms are higher than those of the second-order terms which themselves are much higher than the amplitudes of the third-order terms. The perturbative analysis then follows in a straightforward manner. In other words, the different components of the output signal are derived step by step, i.e., the fundamental terms are calculated first and serve for the determination of the second-order terms. In the same way, both the first- and second-order terms are useful for the calculation of the third-order terms. Under these assumptions, (9) can easily be solved for $s_{\omega}, n_{\omega}$, and $\phi_{\omega}$ :

$$
\begin{aligned}
s_{\omega}= & \frac{\left(b_{\omega_{1}}^{\left|m_{1}\right|} b_{\omega_{2}}^{\left|m_{2}\right|}\right)^{k_{m}}}{d(\omega)} \\
& \cdot\left[K_{11}(\omega) Q_{s, \omega}+K_{12}(\omega) Q_{n, \omega}+K_{13}(\omega) Q_{\phi, \omega}\right] \\
n_{\omega}= & \frac{\left(b_{\omega_{1}}^{\left|m_{1}\right|} b_{\omega_{2}}^{\left|m_{2}\right|}\right)^{k_{m}}}{d(\omega)} \\
& \cdot\left[K_{21}(\omega) Q_{s, \omega}+K_{22}(\omega) Q_{n, \omega}+K_{23}(\omega) Q_{\phi, \omega}\right] \\
\phi_{\omega}= & \frac{\left(b_{\omega_{1}}^{\left|m_{1}\right|} b_{\omega_{2}}^{\left|m_{2}\right|}\right)^{k_{m}}}{d(\omega)} \\
& \cdot\left[K_{31}(\omega) Q_{s, \omega}+K_{32}(\omega) Q_{n, \omega}+K_{33}(\omega) Q_{\phi, \omega}\right]
\end{aligned}
$$

in which $d(\omega)=i \omega\left(\operatorname{smi} A-\omega^{2}\right)-\operatorname{tr} A \omega^{2}+\operatorname{det} A$, where the coefficients tr $A$ and smi $A$, respectively, represent the trace of $A$ and the sum of the minors associated with the elements of the main diagonal, and $\operatorname{det} A$ is the determinant of $A$. The $K_{i j}(\omega)$ are the elements of the adjoint matrix of $i \omega I+A$, where $I$ is the unit matrix of order 3 .

\section{A. First-Order Solutions}

The first-order driving terms are given by $(i=1,2)$

$$
\begin{aligned}
& Q_{s, \omega_{i}}=0 \\
& Q_{n, \omega_{i}}=j_{\omega_{i}} \\
& Q_{\phi, \omega_{i}}=0 .
\end{aligned}
$$

By inserting (13)-(15) into (10)-(12), the first-order solutions can be deduced and expressed as follows:

$$
\begin{aligned}
s_{\omega_{i}} & =\frac{K_{12}\left(\omega_{i}\right)}{d\left(\omega_{i}\right)} j_{\omega_{i}} \\
n_{\omega_{i}} & =u_{\omega_{i}} s_{\omega_{i}} \\
\phi_{\omega_{i}} & =v_{\omega_{i}} s_{\omega_{i}}
\end{aligned}
$$

with

$$
\begin{aligned}
& u_{\omega_{i}}=\frac{K_{22}\left(\omega_{i}\right)}{K_{12}\left(\omega_{i}\right)} \\
& v_{\omega_{i}}=\frac{K_{32}\left(\omega_{i}\right)}{K_{12}\left(\omega_{i}\right)}
\end{aligned}
$$

\section{B. Second-Order Solutions}

The second-order distortions yield distortion products at $2 \omega_{i}$ $(i=1,2)$ and $\omega_{2} \pm \omega_{1}$.

The complex amplitudes of the second harmonics are obtained from (10) to (12) and expressed as

$$
\begin{aligned}
& s_{2 \omega_{i}}=t_{2 \omega_{i}} s_{\omega_{i}}^{2} \\
& n_{2 \omega_{i}}=u_{2 \omega_{i}} s_{\omega_{i}}^{2} \\
& \phi_{2 \omega_{i}}=v_{2 \omega_{i}} s_{\omega_{i}}^{2}
\end{aligned}
$$

with

$$
\begin{aligned}
t_{2 \omega_{i}}=\frac{1}{d\left(2 \omega_{i}\right)}[ & K_{11}\left(2 \omega_{i}\right) Q_{s, 2 \omega_{i}}+K_{12}\left(2 \omega_{i}\right) Q_{n, 2 \omega_{i}} \\
& \left.+K_{13}\left(2 \omega_{i}\right) Q_{\phi, 2 \omega_{i}}\right] \\
u_{2 \omega_{i}}=\frac{1}{d\left(2 \omega_{i}\right)}[ & K_{21}\left(2 \omega_{i}\right) Q_{s, 2 \omega_{i}}+K_{22}\left(2 \omega_{i}\right) Q_{n, 2 \omega_{i}} \\
& \left.+K_{23}\left(2 \omega_{i}\right) Q_{\phi, 2 \omega_{i}}\right] \\
v_{2 \omega_{i}}=\frac{1}{d\left(2 \omega_{i}\right)}[ & K_{31}\left(2 \omega_{i}\right) Q_{s, 2 \omega_{i}}+K_{32}\left(2 \omega_{i}\right) Q_{n, 2 \omega_{i}} \\
& \left.+K_{33}\left(2 \omega_{i}\right) Q_{\phi, 2 \omega_{i}}\right]
\end{aligned}
$$

where the driving terms are given below:

$$
\begin{aligned}
Q_{s, 2 \omega_{i}}= & -\frac{1}{2}\left[\varepsilon_{n} n_{\mathrm{th}} \gamma\left(n_{\mathrm{th}} n_{0}+1-n_{\mathrm{th}}\right)+\frac{1}{4} \tau_{p} \rho_{0} \gamma \cos \theta_{0}\right] \\
& +\frac{1}{2} n_{\mathrm{th}} \gamma\left(1-2 \varepsilon_{n} s_{0}\right) u_{\omega_{i}} \\
& -\frac{\tau_{p} \rho_{0} \gamma}{8 s_{0}}\left(\cos \theta_{0} v_{\omega_{i}}^{2}+2 \sin \theta_{0} v_{\omega_{i}}\right) \\
Q_{n, 2 \omega_{i}}= & -\frac{1}{2}\left(1-2 \varepsilon_{n} s_{0}\right) u_{\omega_{i}} \\
& +\frac{\varepsilon_{n}}{2 n_{\mathrm{th}}}\left(n_{\mathrm{th}} n_{0}+1-n_{\mathrm{th}}\right) \\
Q_{\phi, 2 \omega_{i}}= & -\frac{1}{2} \varepsilon_{n} \alpha n_{\mathrm{th}} \gamma s_{0} u_{\omega_{i}} \\
& -\frac{\tau_{p} \rho_{0} \gamma}{8 s_{0}}\left(3 \sin \theta_{0}-\sin \theta_{0} v_{\omega_{i}}^{2}-2 \cos \theta_{0} v_{\omega_{i}}\right)
\end{aligned}
$$


The complex amplitudes of the terms corresponding to $\Delta \omega$ can be deduced from (10)-(12) and expressed as follows:

$$
\begin{aligned}
& s_{\Delta \omega}=t_{\Delta \omega} s_{\omega_{1}}^{*} s_{\omega_{2}} \\
& n_{\Delta \omega}=u_{\Delta \omega} s_{\omega_{1}}^{*} s_{\omega_{2}} \\
& \phi_{\Delta \omega}=v_{\Delta \omega} s_{\omega_{1}}^{*} s_{\omega_{2}}
\end{aligned}
$$

with

$$
\begin{gathered}
t_{\Delta \omega}=\frac{1}{d(\Delta \omega)}\left[K_{11}(\Delta \omega) Q_{s, \Delta \omega}+K_{12}(\Delta \omega) Q_{n, \Delta \omega}\right. \\
\left.+K_{13}(\Delta \omega) Q_{\phi, \Delta \omega}\right] \\
\begin{aligned}
u_{\Delta \omega}=\frac{1}{d(\Delta \omega)}[ & K_{21}(\Delta \omega) Q_{s, \Delta \omega}+K_{22}(\Delta \omega) Q_{n, \Delta \omega} \\
& \left.+K_{23}(\Delta \omega) Q_{\phi, \Delta \omega}\right]
\end{aligned} \\
\begin{aligned}
v_{\Delta \omega}=\frac{1}{d(\Delta \omega)}[ & K_{31}(\Delta \omega) Q_{s, \Delta \omega}+K_{32}(\Delta \omega) Q_{n, \Delta \omega} \\
+ & \left.K_{33}(\Delta \omega) Q_{\phi, \Delta \omega}\right]
\end{aligned}
\end{gathered}
$$

where the following driving terms must be considered:

$$
\begin{aligned}
Q_{s, \Delta \omega}= & -\varepsilon_{n} \gamma\left(n_{\mathrm{th}} n_{0}+1-n_{\mathrm{th}}\right) \\
& +\frac{1}{2} n_{\mathrm{th}} \gamma\left(1-2 \varepsilon_{n} s_{0}\right)\left(u_{\omega_{1}}^{*}+u_{\omega_{2}}\right) \\
& -\frac{1}{4 s_{0}} \tau_{p} \rho_{0} \gamma\left[\cos \theta_{0}\left(1+v_{\omega_{1}}^{*} v_{\omega_{2}}\right)\right. \\
& \left.+\sin \theta_{0}\left(v_{\omega_{1}}^{*}+v_{\omega_{2}}\right)\right] \\
Q_{n, \Delta \omega}= & -\frac{1}{2}\left(1-2 \varepsilon_{n} s_{0}\right)\left(u_{\omega_{1}}^{*}+u_{\omega_{2}}\right) \\
& +\frac{\varepsilon_{n}}{n_{\mathrm{th}}}\left(n_{\mathrm{th}} n_{0}+1-n_{\mathrm{th}}\right) \\
Q_{\phi, \Delta \omega}= & -\frac{1}{2} \varepsilon_{n} \alpha n_{\mathrm{th}} \gamma s_{0}\left(u_{\omega_{1}}^{*}+u_{\omega_{2}}\right) \\
& -\frac{\tau_{p} \rho_{0} \gamma}{4 s_{0}}\left[3 \sin \theta_{0}-\sin \theta_{0} v_{\omega_{1}}^{*} v_{\omega_{2}}\right. \\
& \left.-\cos \theta_{0}\left(v_{\omega_{1}}^{*}+v_{\omega_{2}}\right)\right] .
\end{aligned}
$$

Relation (10) yields the complex amplitude of the term at $\omega_{I I}$, corresponding to photons in (39), shown at the bottom of the page, with

$$
\begin{aligned}
Q_{s, \omega_{I I}}= & -\varepsilon_{n} \gamma\left(n_{\mathrm{th}} n_{0}+1-n_{\mathrm{th}}\right) \\
& +\frac{1}{2} n_{\mathrm{th}} \gamma\left(1-2 \varepsilon_{n} s_{0}\right)\left(u_{\omega_{1}}+u_{\omega_{2}}\right) \\
& -\frac{\tau_{p} \rho_{0} \gamma}{4 s_{0}}\left[\cos \theta_{0}\left(1+v_{\omega_{1}} v_{\omega_{2}}\right)\right. \\
& \left.+\sin \theta_{0}\left(v_{\omega_{1}}+v_{\omega_{2}}\right)\right] \\
Q_{n, \omega_{I I}}= & -\frac{1}{2}\left(1-2 \varepsilon_{n} s_{0}\right)\left(u_{\omega_{1}}+u_{\omega_{2}}\right) \\
& +\frac{\varepsilon_{n}}{n_{\mathrm{th}}}\left(n_{\mathrm{th}} n_{0}+1-n_{\mathrm{th}}\right)
\end{aligned}
$$

$$
\begin{aligned}
Q_{\phi, \omega_{I I}}= & \frac{1}{2} \varepsilon_{n} \alpha n_{\mathrm{th}} \gamma s_{0}\left(u_{\omega_{1}}+u_{\omega_{2}}\right) \\
& -\frac{\tau_{p} \rho_{0} \gamma}{4 s_{0}}\left[3 \sin \theta_{0}-\sin \theta_{0} v_{\omega_{1}} v_{\omega_{2}}\right. \\
& \left.\quad-\cos \theta_{0}\left(v_{\omega_{1}}+v_{\omega_{2}}\right)\right]
\end{aligned}
$$

\section{Third-Order Solutions}

The third-order spurious frequencies generated under twotone modulation are the third-order harmonics $3 \omega_{i}$ and the third-order IMP's at $2 \omega_{1} \pm \omega_{2}, 2 \omega_{2} \pm \omega_{1}$. Here, only the product $2 \omega_{1}-\omega_{2}$, that we shall denote by $\omega_{I I I}$, will be studied.

Relation (10) gives the amplitude of the third-order intermodulation term corresponding to photons in (43), shown at the bottom of the page, with

$$
\begin{aligned}
& Q_{s, \omega_{I I I}}-\left[\varepsilon_{n} \gamma\left(n_{\mathrm{th}} n_{0}+1-n_{\mathrm{th}}\right)+\frac{1}{4 s_{0}} \tau_{p} \rho_{0} \cos \theta_{0}\right] \\
& \cdot\left(t_{2 \omega_{1}}+t_{\Delta \omega}^{*}\right)+\frac{n_{\mathrm{th}} \gamma}{2}\left(1-2 \varepsilon_{n} s_{0}\right) \\
& \cdot\left(u_{2 \omega_{1}}+u_{\omega_{2}}^{*} t_{2 \omega_{1}}+u_{\Delta \omega}^{*}+u_{\omega_{1}} t_{\Delta \omega}^{*}\right) \\
&-\frac{1}{4} \varepsilon_{n} n_{\mathrm{th}} \gamma\left(2 u_{\omega_{1}}+u_{\omega_{2}}^{*}\right) \\
&-\frac{\tau_{p} \rho_{0} \gamma}{4 s_{0}}\left[\cos \theta_{0}\left(v_{2 \omega_{1}} v_{\omega_{2}}^{*}+v_{\omega_{1}} v_{\Delta \omega}^{*}\right)\right. \\
&\left.\quad+\sin \theta_{0}\left(v_{2 \omega_{1}}+v_{\omega_{2}}^{*} t_{2 \omega_{1}}+v_{\Delta \omega}^{*}+v_{\omega_{1}} t_{\Delta \omega}^{*}\right)\right] \\
&+\frac{\tau_{p} \rho_{0} \gamma}{32 s_{0}^{2}}\left[3 \cos \theta_{0}+\sin \theta_{0}\left(v_{\omega_{1}}^{2} v_{\omega_{2}}^{*}+2 v_{\omega_{1}}+v_{\omega_{2}}^{*}\right)\right. \\
&\left.\quad-\cos \theta_{0}\left(2 v_{\omega_{1}} v_{\omega_{2}}^{*}+v_{\omega_{1}}^{2}\right)\right] \\
& Q_{n, \omega_{I I I}} \quad-\frac{1}{2}\left(1-2 \varepsilon_{n} s_{0}\right)\left(u_{2 \omega_{1}}+u_{\omega_{2}}^{*} t_{2 \omega_{1}}+u_{\Delta \omega}^{*}+u_{\omega_{1}} t_{\Delta \omega}^{*}\right) \\
& \quad+\frac{\varepsilon_{n}}{n_{\mathrm{th}}}\left(n_{\mathrm{th}} n_{0}+1-n_{\mathrm{th}}\right)\left(t_{2 \omega_{1}}+t_{\Delta \omega}^{*}\right) \\
&+\frac{1}{4} \varepsilon_{n}\left(2 u_{\omega_{1}}+u_{\omega_{2}}^{*}\right) \\
& Q_{\phi, \omega_{I I I}} \quad \frac{1}{2} \varepsilon_{n} \alpha n_{\mathrm{th}} \gamma s_{0}\left(u_{2 \omega_{1}}+u_{\omega_{2}}^{*} t_{2 \omega_{1}}+u_{\Delta \omega}^{*}+u_{\omega_{1}} t_{\Delta \omega}^{*}\right) \\
& \quad-\frac{\tau_{p} \rho_{0} \gamma}{4 s_{0}}\left[3 \sin \theta_{0}\left(t_{2 \omega_{1}}+t_{\Delta \omega^{\prime}}^{*}\right)\right. \\
& \quad-\sin \theta_{0}\left(v_{2 \omega_{1}} v_{\omega_{2}}^{*}+v_{\omega_{1}} v_{\Delta \omega}^{*}\right) \\
&\left.\quad-\cos \theta_{0}\left(v_{2 \omega_{1}}+v_{\omega_{2}}^{*} t_{2 \omega_{1}}+v_{\omega_{1}} t_{\Delta \omega}^{*}+v_{\Delta \omega}^{*}\right)\right] \\
& \quad-\frac{\tau_{p} \rho_{0} \gamma}{32 s_{0}^{2}}\left[-15 \sin \theta_{0}-\cos \theta_{0} v_{\omega_{1}}^{2} v_{\omega_{2}}^{*}\right. \\
& \quad+3 \cos \theta_{0}\left(2 v_{\omega_{1}}+v_{\omega_{2}}^{*}\right) \\
&\left.\quad+\sin \theta_{0}\left(2 v_{\omega_{1}} v_{\omega_{2}}^{*}+v_{\omega_{1}}^{2}\right)\right] .
\end{aligned}
$$

$$
s_{\omega_{I I}}=\frac{K_{11}\left(\omega_{I I}\right) Q_{s, \omega_{I I}}+K_{12}\left(\omega_{I I}\right) Q_{n, \omega_{I I}}+K_{13}\left(\omega_{I I}\right) Q_{\phi, \omega_{I I}}}{d\left(\omega_{I I}\right)} s_{\omega_{1}} s_{\omega_{2}}
$$

$$
s_{\omega_{I I I}}=\frac{K_{11}\left(\omega_{I I I}\right) Q_{s, \omega_{I I I}}+K_{12}\left(\omega_{I I I}\right) Q_{n, \omega_{I I I}}+K_{13}\left(\omega_{I I I}\right) Q_{\phi, \omega_{I I I}}}{d\left(\omega_{I I I}\right)} s_{\omega_{1}}^{2} s_{\omega_{2}}^{*}
$$


As mentioned before, we shall report the normalized transfer function $\left|s_{\omega_{1}} / j_{\omega_{1}}\right|$ and the distortion characteristics produced by the second- and third-order IMP's at $f_{I I}$ and $f_{I I I}$, whose effects are measured by the quantities $\left|s_{\omega_{I I}} / s_{\omega_{2}}\right|$ and $\left|s_{\omega_{I I I}} / s_{\omega_{2}}\right|$, respectively. Using (16), (39), and (43) yields (47)-(49), shown at the bottom of the page, where $O M D$ $=\left|s_{\omega_{i}} / s_{0}\right|$ is the optical modulation depth associated with $\omega_{i}$.

Then the second-order IMD is found to vary proportionally with OMD whereas the third-order IMD is proportional to $\mathrm{OMD}^{2}$. This is the same result obtained before for a directly modulated laser without light injection.

From the above relations, it can also be noticed that the intermodulation distortions are dependent on both the carrier frequencies $\left(\omega_{1}, \omega_{2}\right)$ and the particular IMP $\left(\omega_{I I}\right.$ for the second-order IMD and $\omega_{I I I}$ for the third-order). The thirdorder IMD also depends on the second-order harmonic $2 \omega_{1}$ and the second-order IMP at $\Delta \omega=\omega_{2}-\omega_{1}$.

\section{RESUlTS AND DiscUSSION}

Illustrative examples are given in this section. The dynamic characteristics of the injection-locked laser are presented as functions of the modulation frequency. The results are compared with those of the same laser without light injection. The reported curves are obtained for physical parameters adopted to be $\tau_{e}=1 \mathrm{~ns}, n_{\mathrm{th}}=1.9, \gamma=500, T=7 \mathrm{ps}, \beta=0.001$, $\varepsilon=0.01$, and $\alpha=6$. We have also chosen the modulation conditions: $j_{0}=2$ (the bias current is twice the threshold current) and $j_{\omega_{1}}=0.25$ for all curves. The injection-locked regime is illustrated for three injection levels of $-26,-20$, and $-14 \mathrm{~dB}$, corresponding, respectively, to dynamically stable locking ranges of $-30.9 /-12.5 \mathrm{GHz},-40.7 /-12.4 \mathrm{GHz}$, and $-61.8 /-11.1 \mathrm{GHz}$, in which the operating conditions are chosen. The numerical values of the injection parameters are indicated in the figure captions. The case of the transmission of a single channel in a narrow band centered around a high-frequency carrier is considered. Specifically, the laser is supposed to be modulated with two subcarrier signals with closely spaced frequencies $f_{1}=f_{c}+\Delta f / 2$ and $f_{2}=$ $f_{c}-\Delta f / 2$, with $\Delta f=10 \mathrm{MHz}, f_{c}$ being the center frequency of the channel. Equal modulation amplitudes are applied for $f_{1}$ and $f_{2}$. The situation of relatively strong light injection is considered.

The first-order transfer function, also known as modulation response, is reported in Figs. 1 and 2 through different examples showing the influence of detuning and injection level. Depending on the assumed injection parameters, these char-

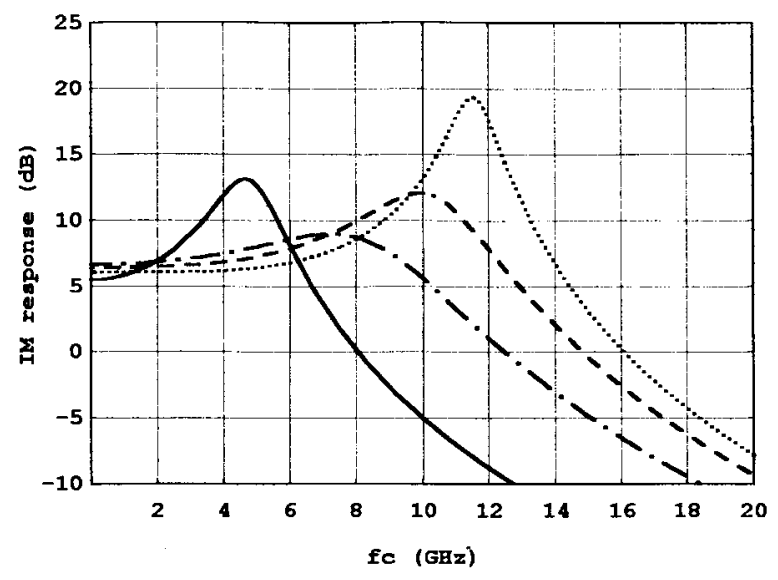

$$
\begin{array}{ll}
\ldots & \text { Noninjected regime }\left(\tau_{r}=0.154 \mathrm{~ns}\right) \\
\ldots- & \left(\Delta \nu_{0}=-35 \mathrm{GH} z, \tau_{r}=0.056 \mathrm{~ns}\right) \\
\ldots \ldots & \left(\Delta \nu_{0}=-25 \mathrm{GHz}, \tau_{r}=0.086 \mathrm{~ns}\right) \\
\ldots . & \left(\Delta \nu_{0}=-15 \mathrm{GHz}, \tau_{r}=0.217 \mathrm{~ns}\right)
\end{array}
$$

Fig. 1. Influence of frequency detuning on the IM response.

acteristics give the first interesting results for the laser under light injection in comparison with the noninjected regime. The changes are observed concerning three important parameters of the modulation, namely the resonance peak height as well as the modulation bandwidth or resonance frequency. From Fig. 1, the resonance peak height is seen to increase when the detuning is increased with the injection level maintained to a constant value. Conversely, increasing the injection level, at a fixed detuning, results in a rapid decrease and broadening of the resonance peak height (Fig. 2). This behavior is well in agreement with the numerical values of the respective damping time constants shown in the captions. The damping time constants, $\tau_{r}$, are obtained by writing that $\omega= \pm \omega_{r}+$ $j \tau_{e} / \tau_{r}$ cancel the $d(\omega)$ function. The $\tau_{r}$ parameter is of great importance for modulation. It is tightly associated with the turn-on transient oscillations of the laser diode and also characterizes the peak height and width of the frequency response and consequently the amount of distortions that might be generated near resonance. The weaker $\tau_{r}$ is, the less pronounced is the resonance peak and the more rapidly damped are the turn-on transient oscillations, and the lower the distortion levels. Hence, the phenomenon of peak height reduction and broadening with higher injection is expected to be the main reason behind reduction of inherent nonlinear distortions. This will be discussed later.

$$
\begin{aligned}
\left|\frac{s_{\omega_{1}}}{j_{\omega_{1}}}\right| & =\frac{\left[\left(a_{12} \omega_{1}\right)^{2}+\left(a_{32} a_{13}-a_{12} a_{33}\right)^{2}\right]^{1 / 2}}{\left|d\left(\omega_{1}\right)\right|} \\
\left|\frac{s_{\omega_{I I}}}{s_{\omega_{2}}}\right| & =O M D s_{0}\left|\frac{K_{11}\left(\omega_{I I}\right) Q_{s, \omega_{I I}}+K_{12}\left(\omega_{I I}\right) Q_{n, \omega_{I I}}+K_{13}\left(\omega_{I I}\right) Q_{\phi, \omega_{I I}}}{d\left(\omega_{I I}\right)}\right| \\
\left|\frac{s_{\omega_{I I I}}}{s_{\omega_{2}}}\right| & =O M D^{2} s_{0}^{2}\left|\frac{K_{11}\left(\omega_{I I I}\right) Q_{s, \omega_{I I I}}+K_{12}\left(\omega_{I I I}\right) Q_{n, \omega_{I I I}}+K_{13}\left(\omega_{I I I}\right) Q_{\phi, \omega_{I I I}}}{d\left(\omega_{I I I}\right)}\right|
\end{aligned}
$$



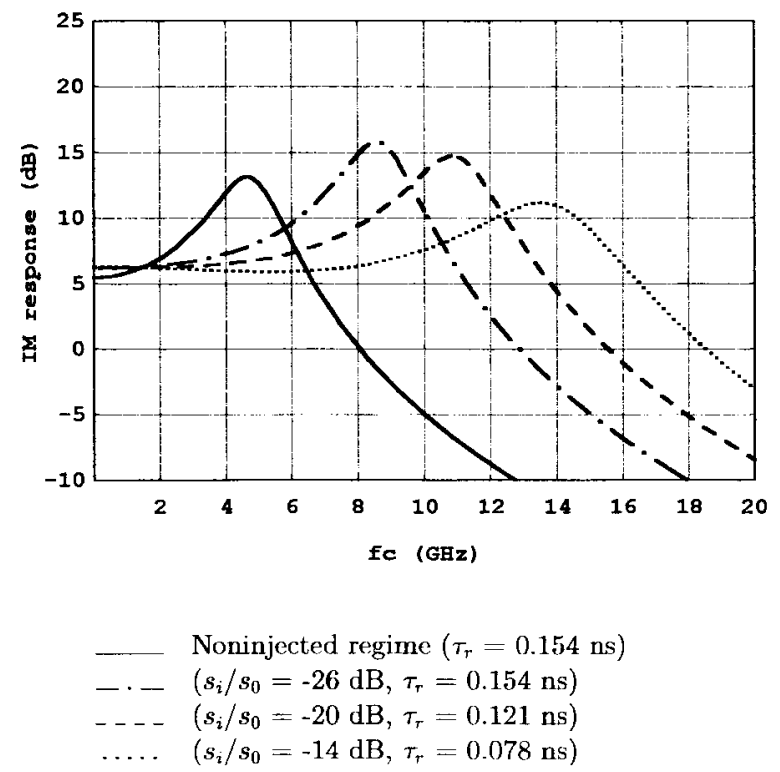

Fig. 2. Influence of injection level on the IM response.

The second change in the frequency response is the increased modulation bandwidth or resonance frequency with either detuning or injection level. On the other hand, the comparison between the injected and free-running regimes shows that the behavior of the laser with light injection can be improved over the same laser under solitary operation. For example, the injection-locked laser with $s_{i} / s_{0}=-14 \mathrm{~dB}$, $\Delta \nu_{0}=-20 \mathrm{GHz}$ (Fig. 2), exhibits a resonance frequency and a damping time constant of about $13.5 \mathrm{GHz}$ and $0.078 \mathrm{~ns}$, respectively, to be compared with the equivalent $4.9 \mathrm{GHz}$ and $0.154 \mathrm{~ns}$ of the same laser under free-running operation. The reason for this increase in resonance frequency or modulation bandwidth with injection has yet not been fully investigated but might be related to the coherent addition of the injected optical field with the slave laser optical field in the slave laser cavity, rather than by the increase in photon density. We have attempted to verify this explanation by adopting the values of the normalized steady-state photon density $s_{0}$ in the freerunning regime to be equal to those of the injection-locked laser, obtained for different injection parameters. We found increased resonance frequency and modulation bandwidth, but to values much less than those observed under light injection. This result agrees with the considered assumption. It can be proved, in mathematical terms, by deriving an explicit expression of either of both quantities. This aspect of the investigation is not carried out here, but could be examined in the future.

Figs. 3 and 4 report the second-order IMD of type $f_{2}+f_{1}$ whereas Figs. 5 and 6 report the third-order IMD at $2 f_{1}-f_{2}$. The distortion levels are plotted as decibels relative to the carrier $(\mathrm{dBc})$ intensity at frequency $f_{2}$. Figs. 3 and 5 describe the influence of frequency detuning upon the second- and third-order IMD's. They are derived by simulation at an injection level of $-20 \mathrm{~dB}$ with varying detuning of $-35,-25$, and $-15 \mathrm{GHz}$. On the other hand, Figs. 4 and 6 show the influence of injection level, corresponding to a fixed detuning

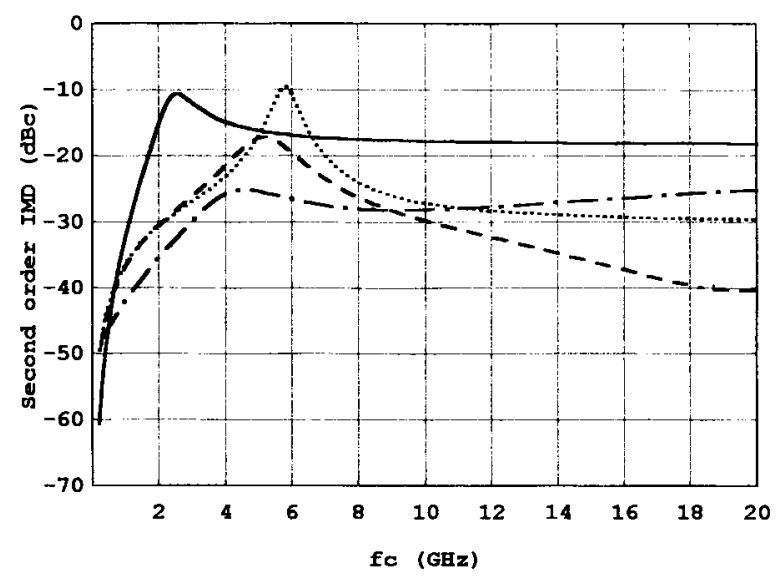

$$
\begin{array}{ll}
\ldots & \text { Noninjected regime }\left(\tau_{\tau}=0.154 \mathrm{~ns}\right) \\
\cdots \cdot- & \left(\Delta \nu_{0}=-35 \mathrm{GHz}, \tau_{\tau}=0.056 \mathrm{~ns}\right) \\
-\cdots & \left(\Delta \nu_{0}=-25 \mathrm{GHz}, \tau_{\tau}=0.086 \mathrm{~ns}\right) \\
\ldots . & \left(\Delta \nu_{0}=-15 \mathrm{GHz}, \tau_{\tau}=0.217 \mathrm{~ns}\right)
\end{array}
$$

Fig. 3. Influence of frequency detuning on the second-order intermodulation product, of type $f_{2}+f_{1}\left(s_{i} / s_{0}=-20 \mathrm{~dB}\right)$.
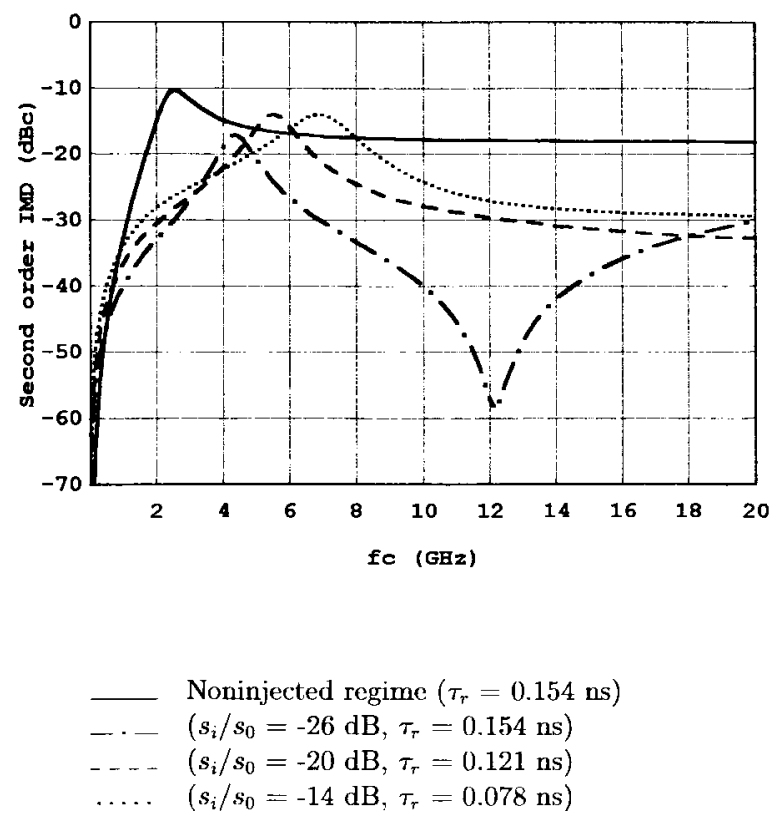

Fig. 4. Influence of injection level on the second-order intermodulation product, of type $f_{2}+f_{1}\left(\Delta \nu_{0}=-20 \mathrm{GHz}\right)$.

of $\Delta \nu_{0}=-20 \mathrm{GHz}$ and varying injection level of $-26,-20$, and $-14 \mathrm{~dB}$. The free-running operation characteristics are shown in each figure for comparison. Curves in Figs. 4-6 are plotted for a constant optical modulation depth, adopted to be a low-frequency OMD given by $O M D=s_{\omega_{i}}(0) / s_{0}$. Practically speaking, this can be achieved by adjusting the drive amplitude at different frequencies so that the first harmonic response remains constant (i.e., prefilter the modulation current).

As can be seen from Figs. 3-6, for the solitary laser, the second- and third-order distortions peak near the half resonance frequency $f_{r}$. The third-order distortion also shows 

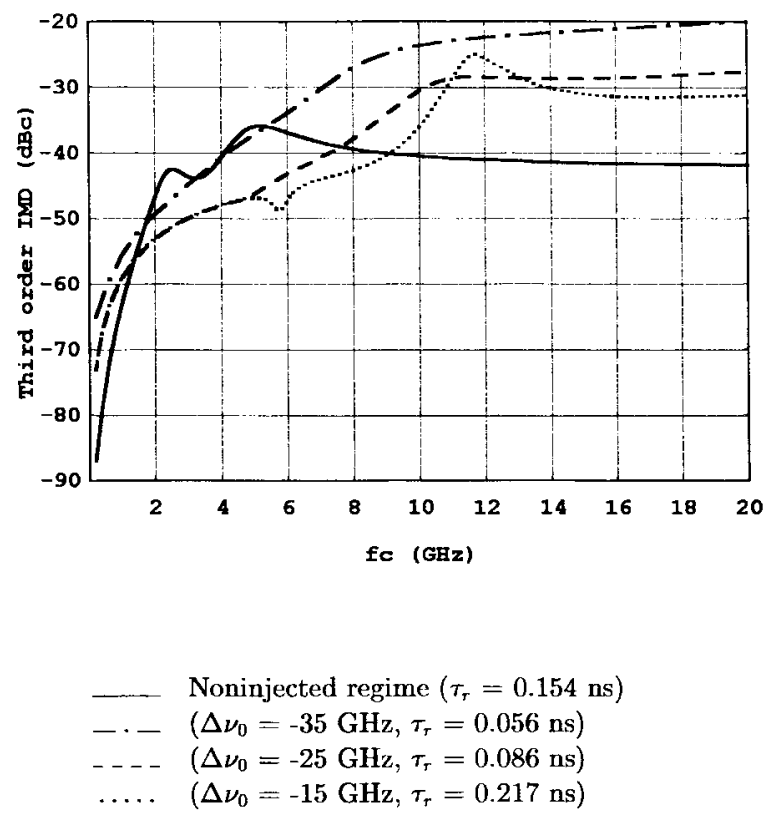

Fig. 5. Influence of frequency detuning on the third-order intermodulation product, of type $2 f_{1}-f_{2}\left(s_{i} / s_{0}=-20 \mathrm{~dB}\right)$.
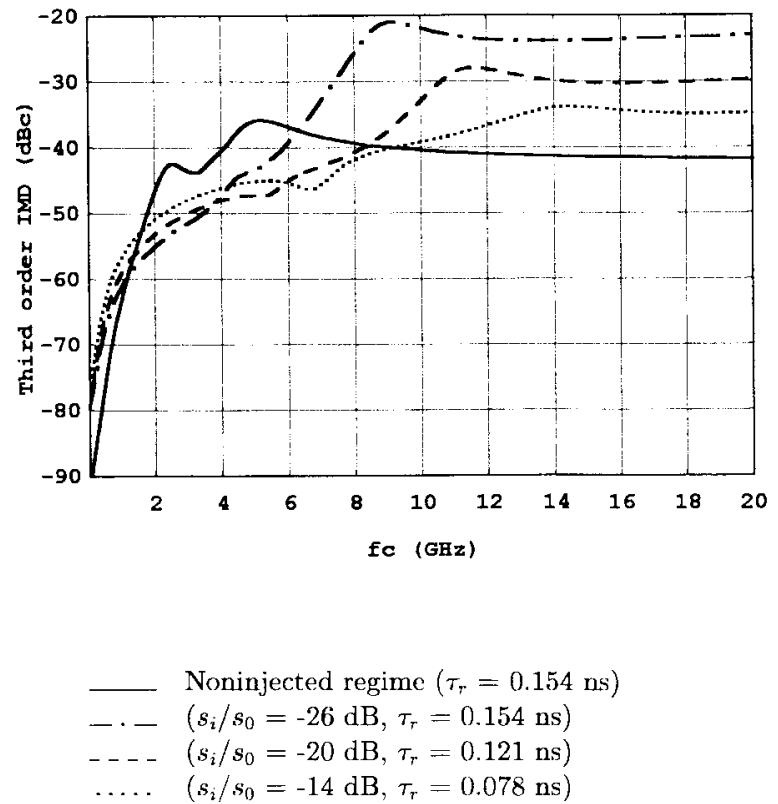

Fig. 6. Influence of injection level on the third-order intermodulation product, of type $2 f_{1}-f_{2}\left(\Delta \nu_{0}=-20 \mathrm{GHz}\right)$

a maximum and a dip at frequencies near $f_{r}$ and $f_{r} / \sqrt{2}$, respectively. These findings were known before [3], [5]. For the injection-locked regime, the same properties are, generally, kept, except that the maxima and dips are plus or less extended, depending of both injection level and detuning. The secondorder distortion is also likely to exhibit a dip at frequencies much higher than the resonance frequency. We notice that the observed dips do not appear for some values of injection level and detuning.

Fig. 4 shows that as long as the laser operates with a constant frequency detuning, the influence of injection level on the second-order IMD is a little on the low-frequency side, roughly

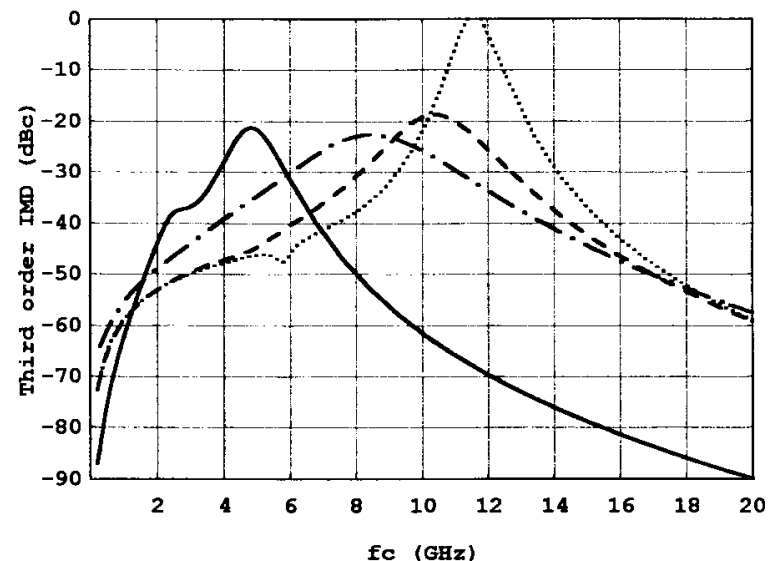

$$
\begin{array}{ll}
- & \text { Noninjected regime }\left(\tau_{r}=0.154 \mathrm{~ns}\right) \\
-\cdot- & \left(\Delta \nu_{0}=-35 \mathrm{GHz}, \tau_{\tau}=0.056 \mathrm{~ns}\right) \\
--- & \left(\Delta \nu_{0}=-25 \mathrm{GHz}, \tau_{r}=0.086 \mathrm{~ns}\right) \\
\ldots . & \left(\Delta \nu_{0}=-15 \mathrm{GHz}, \tau_{r}=0.217 \mathrm{~ns}\right)
\end{array}
$$

Fig. 7. Influence of frequency detuning on the third-order intermodulation product of type $2 f_{1}-f_{2}$, for a constant modulating current and a fixed injection level $s_{i} / s_{0}=-20 \mathrm{~dB}$.

for modulation frequencies lower than the half resonance frequency. However, inspection of the same figure shows that the second-order distortion increases in the high-frequency side with increasing injection level. It is also seen (Fig. 3) that higher detuning generally increases the levels of second-order IMD in the low-frequency side where, practically, the laser is to be modulated. Conversely, Figs. 5 and 6 show that the thirdorder distortion tends to decrease with an increasing frequency detuning or injection level. More important is the comparison between the free-running and injection-locked regimes. Fig. 3 shows that an injection-locking with $s_{i} / s_{0}=-20 \mathrm{~dB}$ and $\Delta \nu_{0}=-35 \mathrm{GHz}$ might reduce the second-order distortion of more than $7 \mathrm{dBc}$ for modulation frequencies above $1 \mathrm{GHz}$. For the same set of parameters, the reduction in second-order IMD might attain $20 \mathrm{dBc}$ or more in the half resonance frequency region of the solitary laser. A reduction of the third-order distortion is also obtained by light injection over $2-8 \mathrm{GHz}$, which corresponds to the maximum distortion regions of the solitary laser. An interesting fact is that second- and thirdorder distortions can be improved at the same time, even if a suitable compromise must be chosen between second- and third-order IMD's.

The resonance frequency enhancement with injection as well as the broadening and reduction of the resonance peak height are the main reasons behind reduction in nonlinear distortion with injection. This connection has not been highlighted enough through the above distortion characteristics since curves are plotted for a constant OMD (i.e., flat frequency response). Hence, the $t_{r}$ values reported in Figs. 3-6 have, in fact, little significance for these curves but are principally indicative. To show the impact of the damping time constant upon the characteristics, an example is given in Fig. 7, corresponding to the third-order IMD plotted for a constant modulating current instead of constant OMD. As 
expected, in agreement with higher $t_{r}$, the resonance peak height of the third-order IMD increases. On the other hand, the distortion levels are seen to be much more reduced in the resonance frequency region of the free-running operation regime. For $\Delta \nu_{0}=-25$ or $-15 \mathrm{GHz}$, the obtained reduction in third-order IMD is more than $25 \mathrm{~dB}$ around $5 \mathrm{GHz}$ against $10 \mathrm{~dB}$ for the constant OMD case.

Even if experiment is required, these results show that the injection-locking technique should be expected to significantly improve the dynamic behavior of analog modulated subcarrier systems operating well into the microwave frequency range. It can be mentioned, however, that a great care must be taken when modulating a laser under relatively strong light injection. As shown in [18], only a part of the total locking range is dynamically stable. To operate the laser in this range and avoid an eventually severe instability, a suitable choice of the parameter values is necessary. It is even preferable to choose them far enough from the high- and low-frequency ends of the dynamically stable locking range to prevent any problem that might result from a possible temperature rise in the medium.

To this end, it has to be noticed that the injection-locking technique is not very easy to be implemented using conventional semiconductor lasers because of their large spectral linewidth and insufficient frequency stability. In addition, to measure a precise interaction with the injected laser beam, an optical isolator would have to be equipped in order to avoid undesired feedback into the master laser. All this to some extent limits the practical applications at present, although some commercially high-performance lasers are readily injectionlocked systems. But, in spite of the difficulties of applying the technique, injection-locking may be expected to find a much greater interest with the availability of new laser structures or integrated modules having a good frequency stability and narrow linewidth.

\section{CONCLUSION}

We have presented a detailed analysis of the dynamic characteristic of a semiconductor laser under injection-locking. The used theory is based on three coupled differential nonlinear equations for the normalized photon and electron densities of a laser with a $\mathrm{CW}$ coherent light injection. The equations used include the important carrier density dependence of the refractive index (described by the linewidth enhancement factor $\alpha$ ) and the gain compression (expressed by $\varepsilon$ parameter).

This paper shows that when a free-running laser is injectionlocked, one can significantly improve the dynamic behavior and thus reduce the influence of the undesirable components of the output signal, over a wide frequency range. In addition, we demonstrate that injection-locking can be used to reduce the resonance peak and increase the modulation bandwidth of the injected laser. These findings are very attractive in connection with high-frequency applications of laser diodes. It appears that the obtained results are strongly sensitive to the choice of parameters, particularly the injection level and frequency detuning between the master and slave lasers. Larger improvements are possible than those presented here, by suitably adjusting the parameter values. To substantially reduce the second-order distortion, low values of injection level and detuning must be chosen. On the contrary, high values of both parameters are required to reduce the thirdorder distortion. Thus, to obtain a simultaneous reduction in second- and third-order distortion, a compromise has to be adopted.

It is appropriate to note that this work is achieved in the case where two sinusoidal currents of different frequencies $f_{1}$ and $f_{2}$ are applied to the laser diode. In practice, several channels are simultaneously transmitted, for example, when subcarrier multiplexing (SCM) systems are used. Increasing the number of channels from two to three or more greatly increases the number of spurious signals generated. In addition to the $2 f_{1}-f_{2}$ type of intermodulation, the nonlinear mixing of three frequencies together produces another third-order IMP of $f_{1}+f_{2}-f_{3}$ type. For a solitary laser, the presence of these spurious signals and their relative amplitudes have been verified in an actual multichannel lightwave system using a spectrum analyzer [20]. The third-order distortion was found to be dominated by the three-tone products at $f_{1}+f_{2}-f_{3}$, which result in $6-\mathrm{dBc}$ higher distortion levels than those produced by the two-tone products. Moreover, the calculation of the distortion caused by the three-tone products under injectionlocking is of great interest. Further work will be undertaken to completely explore the potential of this linearization technique using light injection.

\section{APPENDIX}

The elements $a_{i j}$ in (9) are given by

$$
\begin{aligned}
& a_{11}=-\gamma\left[\left(n_{\mathrm{th}} n_{0}+1-n_{\mathrm{th}}\right)\left(1-2 \varepsilon_{n} s_{0}\right)-1+\rho_{0} \tau_{p} \cos \theta_{0}\right] \\
& a_{12}=-\gamma n_{\mathrm{th}}\left[s_{0}\left(1-\varepsilon_{n} s_{0}\right)+\beta\right] \\
& a_{13}=\gamma \rho_{0} \tau_{p} \sin \theta_{0} \\
& a_{21}=\frac{1}{n_{\mathrm{th}}}\left(n_{\mathrm{th}} n_{0}+1-n_{\mathrm{th}}\right)\left(1-2 \varepsilon_{n} s_{0}\right) \\
& a_{22}=1+s_{0}\left(1-\varepsilon_{n} s_{0}\right) \\
& a_{23}=0 \\
& a_{31}=-\gamma\left[\varepsilon_{n} \alpha s_{0}\left(n_{\mathrm{th}} n_{0}+1-n_{\mathrm{th}}\right)+\rho_{0} \tau_{p} \sin \theta_{0}\right] \\
& a_{32}=\gamma \alpha n_{\mathrm{th}} s_{0}\left(1-\varepsilon_{n} s_{0}\right) \\
& a_{33}=\gamma \rho_{0} \tau_{p} \cos \theta_{0}
\end{aligned}
$$

where $\rho_{0}=T^{-1}\left(s_{i} / s_{0}\right)^{1 / 2}$, the ratio $s_{i} / s_{0}$ being defined as the injection level.

\section{ACKNOWLEDGMENT}

The authors wish to thank J. Debeau and E. Le Coquil from the Centre National d'Etudes des Télécommunications (CNET), Lannion, and F. Ropars from Ecole Nationale d'Ingénieurs de Brest (ENIB), for helpful discussions.

\section{REFERENCES}

[1] R. Olshansky, V. A. Lanzisera, and P. M. Hill, "Subcarrier multiplexed lightwave systems for broadband distribution," J. Lightwave Technol., vol. 7, pp. 1329-1342, 1989.

[2] K. Y. Lau and A. Yariv, "Intermodulation distortion in a directly modulated semiconductor injection laser," Appl. Phys. Lett., vol. 45, pp. 1034-1036, 1984. 
[3] T. E. Darcie, R. S. Tucker, and G. J. Sullivan, "Intermodulation and harmonic distortion in InGaAsP lasers," Electron. Lett., vol. 21, pp. 665-666, 1985

[4] T. E. Darcie, R. S. Tucker, and G. J. Sullivan, "Intermodulation and harmonic distortion in InGaAsP lasers," Electron. Lett., vol. 22, p. 619, 1986.

[5] J. Wang, M. K. Haldar, and F. V. C. Mendis, "Formula for two-carrier third-order intermodulation distortion in semiconductor laser diodes," Electron. Lett., vol. 29, pp. 1341-1343, 1993.

[6] W. I. Way, "Large signal nonlinear distortion prediction for a singlemode laser diode under microwave intensity modulation," J. Lightwave Technol., vol. LT-5, pp. 305-315, 1987.

[7] D. E. Dodds and M. J. Sieben, "Fabry-Perot laser diode modeling," IEEE Photon. Technol. Lett., vol. 7, pp. 254-256, 1995.

[8] T. K. Biswas and W. F. McGee, "Volterra series analysis of semiconductor laser diode," IEEE Photon. Technol. Lett., vol. 3, pp. 706-708, 1991.

[9] L. Hassine, Z. Toffano, F. Lamnabhi-Lagarrigue, A. Destrez, and C. Birocheau, "Volterra functional series expansions for semiconductor lasers under modulation," IEEE J. Quantum Electron., vol. 30, pp. 918-928, 1994.

[10] W. Harth, "Large-signal direct modulation of injection lasers," Electron. Lett., vol. 9, pp. 532-533, 1973.

[11] J. Le Bihan and G. Yabre, "FM and IM intermodulation distortions in directly modulated single-mode semiconductor lasers," IEEE J. Quantum Electron., vol. 30, pp. 899-904, 1994.

[12] A. Van De Grijp, J. C. Koopman, L. J. Meuleman, A. J. A. Nicia, E. Roza, and J. H. C. Van Heuven, "Novel electro-optical feedback technique for noise and distortion reduction in high-quality analogue optical transmission video signals," Electron. Lett., vol. 17, pp. 361-362, 1981.

[13] L. S. Fock and R. S. Tucker, "Simultaneous reduction of intensity noise and distortion in semiconductor lasers by feedforward compensation," Electron. Lett., vol. 27, no. 14, pp. 1297-1298, 1991.

[14] R. B. Childs and V. A. O'Byrne, "Predistortion linearization of directly modulated DFB lasers and external modulators for AM video transmission," in Tech. Dig. Optical Fiber Communications Conf., San Francisco, CA, 1990, paper VH6.

[15] L. Li and K. Petermann, "Small-signal analysis of optical-frequency conversion in an injection-locked semiconductor laser," IEEE J. Quantum Electron., vol. 30, pp. 43-48, 1994.

[16] J. M. Liu and T. B. Simpson, "Characterization of fundamental parameters of a semiconductor laser with an injected optical probe," IEEE Photon. Technol. Lett., vol. 4, pp. 380-382, 1993.

[17] S. Mohrdiek, H. Burkhard, and H. Walter, "Chirp reduction of directly modulated semiconductor lasers at $10 \mathrm{~Gb} / \mathrm{s}$ by strong $\mathrm{CW}$ light injection," J. Lightwave Technol., vol. 12, pp. 418-424, 1994.
[18] G. Yabre, "Effect of relatively strong light injection on the chirp-topower-ratio and the 3-dB bandwidth of directly modulated semiconductor lasers," J. Lightwave Technol., vol. 14, pp. 2367-2373, Oct. 1996.

[19] L. Goldberg, H. F. Taylor, J. F. Weller, and D. M. Bloom, "Microwave signal generation with injection-locked laser diodes," Electron. Lett., vol. 19 , pp. 491-493, 1983.

[20] P. Iannone and T. E. Darcie, "Multichannel intermodulation distortion in high-speed GaInAsP lasers," Electron. Lett., vol. 23, pp. 1361-1362, 1987.

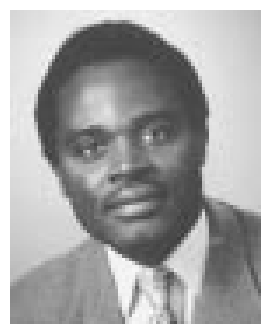

Gnitabouré Yabre was born in Boutaya-P/Zabré, Burkina Faso, in 1962. He received the DEA degree in Electronics and the Doctorate degree in optronics, both from the University of Brest, France, in 1989 and 1993, respectively.

Since 1989, he has been working in the RESO Laboratory of the Ecole Nationale d'Ingénieurs de Brest, France. His current research interests include semiconductor laser nonlinearities and optical communication systems.

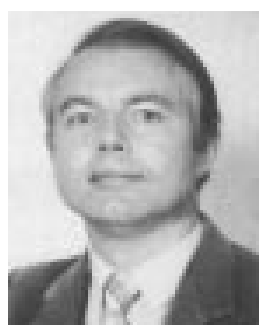

Jean Le Bihan (M'94-SM'95-F'97) was born in 1947. He studied at the Ecole Normale Supérieure, Cachan, and at the Universities of Orsay and Paris. He received the degree of Docteur d'Etat ès Sciences Physiques from Brest University, France, for his work dealing with application of $\mathrm{Z}$ transform to transient analysis of distributed circuits.

After receiving the Aggregation of Applied Physics, he was appointed to Brest Institute of Technology and, in addition, joined the Laboratory of Electronics of Brest University. Later, he became an Assistant Professor of Electronic Engineering at Brest University. From 1986 to 1988, he worked as a Visiting Researcher, first in CAD of MMIC at Thomson-DAG, Corbeville, Orsay, France, then in Optoelectronics at CNET, Lannion, France. Since 1988, as a Professor at the Ecole Nationale d'Ingénieurs de Brest, Brest, France, he has been the head of RES Laboratory. At present, his research interests are mainly in the area of semiconductor lasers for optical communication systems. 